\title{
Diagnosis of Langerhans Cell Histiocytosis on Fine Needle Aspiration Cytology: A Case Report and Review of the Cytology Literature
}

\author{
Neeta Kumar, ${ }^{1}$ Shahin Sayed, ${ }^{1}$ and Sudhir Vinayak ${ }^{2}$ \\ ${ }^{1}$ Department of Pathology, Aga Khan University Hospital, Third Parklands Avenue, P.O. Box 30270, GPO 00100, Nairobi, Kenya \\ ${ }^{2}$ Department of Radiology, Aga Khan University Hospital, Third Parklands Avenue, P.O. Box 30270, GPO 00100, Nairobi, Kenya
}

Correspondence should be addressed to Neeta Kumar, kumar_neeta@hotmail.com

Received 3 October 2010; Accepted 20 December 2010

Academic Editor: Z. Baloch

Copyright (c) 2011 Neeta Kumar et al. This is an open access article distributed under the Creative Commons Attribution License, which permits unrestricted use, distribution, and reproduction in any medium, provided the original work is properly cited.

\begin{abstract}
A case of multifocal Langerhans cell histiocytosis in a two-year-old child is presented where fine needle aspiration was helpful in achieving a rapid and accurate diagnosis in an appropriate clinical and radiological setting. This can avoid unnecessary biopsy and guide the management especially where access to histopathology is limited. The highly characteristic common and rare cytological features are highlighted with focus on differential diagnoses and causes of pitfalls.
\end{abstract}

\section{Introduction}

Langerhans cell histiocytosis ( $\mathrm{LCH}$ ) is a rare disease affecting predominantly children. It can present as a solitary lesion requiring no treatment or as a multisystem, life-threatening disorder necessitating aggressive therapy [1].

We present a case of LCH in a child where fine needle aspiration (FNA) was helpful in establishing a rapid and correct diagnosis in correlation with radiology. The purpose is to highlight common and rare cytological features. This will add to the pathologist's confidence in rendering a rapid and accurate cytologic diagnosis, avoid unnecessary biopsy and guide appropriate management. This is especially valuable in a setting where cytopathologist expertise may not be easily available and histopathology services are located only in big cities and are inaccessible to patients in rural areas due to the long distance and high cost involved.

\section{Case Report}

A two-year-old female child presented to the outreach centre of our university hospital with swellings on right frontal and occipital regions of skull for the last one year. On examination, these were fluctuant, ill-defined soft tissue masses which measured $2 \times 2$ and $3 \times 3 \mathrm{cms}$, respectively. In addition, a cervical lymph node was palpable on the left side measuring $1 \times 1 \mathrm{cms}$. It was firm, tender, and slightly mobile. The patient had no fever or loss of weight. The liver and spleen were not palpable.

Peripheral blood film showed microcytic hypochromic anemia. Hemoglobin was $10 \mathrm{gm} / \mathrm{dL}$. Differential count showed $19 \%$ monocytosis with $38.3 \%$ neutrophils, $38.9 \%$ lymphocytes, $2.5 \%$ eosinophils, and $1.3 \%$ basophils. Platelet count was normal. The initial clinical impression favored a malignant lesion. The patient was referred for FNA.

FNA from lymph node yielded whitish aspirate. FNA from right frontal and occipital masses yielded $0.5 \mathrm{~mL}$ and $1 \mathrm{~mL}$ hemorrhagic fluid, respectively. The fluid was centrifuged to make smears from the sediment. Ethanolfixed smears and air-dried smears were prepared and stained with Papanicolaou and Giemsa method, respectively. The remaining sediment was processed to make cell block for immunochemistry.

Smears were highly cellular and showed numerous atypical histiocytes as the predominant cell type scattered singly and in loosely cohesive clusters. These were admixed with a polymorphic population of eosinophils, neutrophils, lymphocytes, plasma cells, foamy histiocytes, and multinucleated reactive histiocytic giant cells (Figure 1). Smears from both the swellings in the skull and cervical lymph node 
were morphologically similar except that atypical histiocytes were less in number and eosinophils were more abundant in smears from lymph node as compared to smears from skull lesions.

The atypical histiocytes were large cells with moderate to abundant, pale blue cytoplasm and an eccentric or central round to oval, vesicular nuclei. Prominent nuclear indentations and grooves (with a coffee bean appearance) were observed which were best seen in Papanicolaou stain (Figure 2). Some showed intranuclear pseudoinclusions. Nucleoli were absent. These cells displayed marked pleomorphism with variation in size and shape of cells and nuclei. Occasional mitoses were seen. Some of these cells showed cytoplasmic processes. Most were mononuclear, and some were binucleate or multinucleated. The multinucleated giant cells had complex folded nuclei similar to mononuclear atypical histiocytes and were easily differentiated from reactive multinucleated histiocytic giant cells.

The multinucleated reactive histiocytic giant cells contained numerous indented vesicular nuclei in abundant cytoplasm. They also contained hemosiderin in smears from skull masses (Figure 1). In addition, many rhomboid and needle-shaped Charcot-Leyden crystals were seen both extracellular (Figure 3(a)) and intracellular in the giant cells (Figure 3(b)). The atypical histiocytes stained positive for both cytoplasmic and nuclear S-100 protein. The cytologic findings were highly suggestive of LCH.

At this point, a plain X-ray was requested which showed two lytic lesions corresponding to occipital and frontal swellings. Subsequently, computerized tomogram (CT) with $3 \mathrm{D}$ reconstruction showed lytic lesions which were clearly demarcated "punched-out" lesions (the classic geographic skull) in frontal and occipital regions. There was associated homogenous soft tissue swelling of the scalp but no breech of the dura. No other systemic involvement was found.

\section{Discussion}

$\mathrm{LCH}$ is a rare disease, and the estimated annual incidence ranges from 0.5 to 5.4 cases per million persons [2]. In the past, the disorder was referred to as histiocytosis $\mathrm{X}$ and had three variants: eosinophilic granuloma, Hand-SchullerChristian disease and Letterer-Siwe syndrome. These three conditions are believed to represent different expressions of the same disorder, now known as LCH $[3,4]$.

An ongoing debate exists over whether this is a reactive or neoplastic process [2]. The disease is characterized by a clonal proliferation of the antigen-presenting dendritic cell called the Langerhans cell (LC) $[5,6]$. The proliferation may be induced by a viral infection, a defect in $\mathrm{T}$ cellmacrophage interaction, and/or a cytokine-driven process mediated by tumor necrosis factor, interleukin 11, and leukemia inhibitory factor $[2,7-10]$.

LCH may occur at any age, although the majority of the cases are diagnosed in children from newborn to 15 years. There is no significant gender difference. The clinical spectrum varies from a solitary lesion, to multifocal unisystem to multisystem lesions with related symptoms. The unifocal form usually involves the bone, often seen in

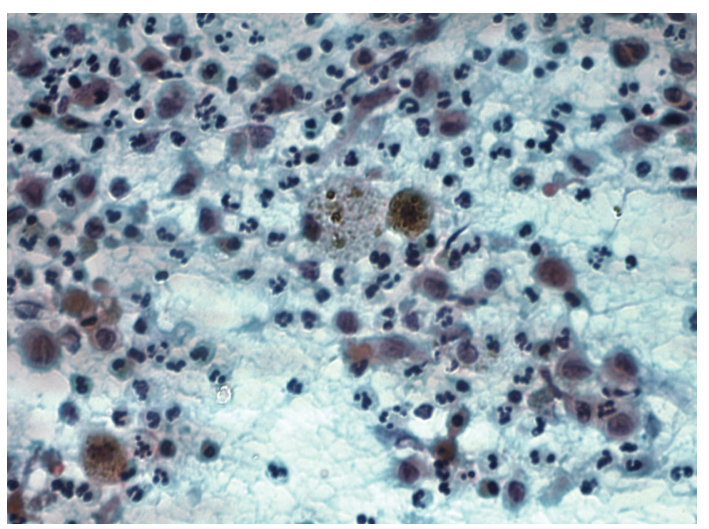

FIGURE 1: FNA smear from frontal mass showing single and loosely clustered Langerhans cells admixed with neutrophils, lymphocytes and, reactive histiocytes. Two foamy macrophages containing hemosiderin are seen in the centre (Papanicolaou stain, original magnification, $\times 400)$.

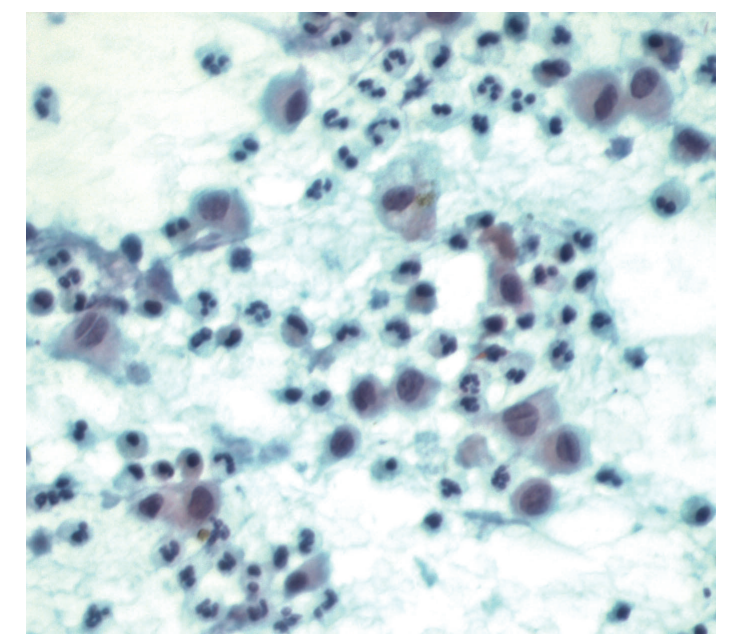

FIGURE 2: Langerhans cells with moderate to abundant cytoplasm and prominent nuclear grooves (Papanicolaou stain, original magnification, $\times 400)$.

children between 5 and 15 years old. Systemic LCH is more common in children under 2 years of age. The multifocal unisystem form almost always occurs in the bone. Any bone can be involved, but more than $50 \%$ of lesions occur in the skull, spine, pelvis, ribs, and mandible. The multifocal multisystem form involves many organs, including the bone, skin, liver, spleen, hematopoietic system, and lymph node $[2,11]$. The lymph node involvement in LCH can be seen as a part of a systemic disease or as a localized lesion, although isolated nodal involvement is rare. Lymph node may also enlarge as a reaction to bone or skin lesions [12].

Traditionally, the diagnosis of LCH is based on hematologic and histologic criteria [2, 4, 13-15]. Enough experience has accumulated in accurate cytological diagnosis of LCH in various body sites on the basis of characteristic cytological features in the presence of appropriate clinical and radiological setting as evident from several case reports 


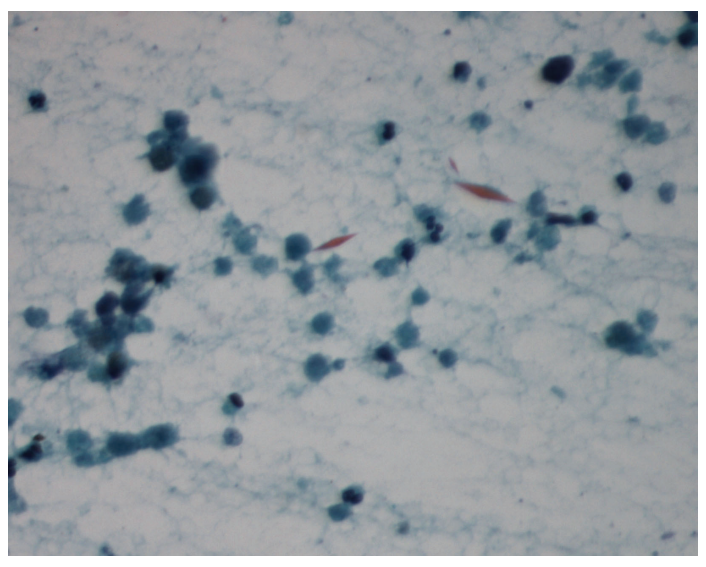

(a)

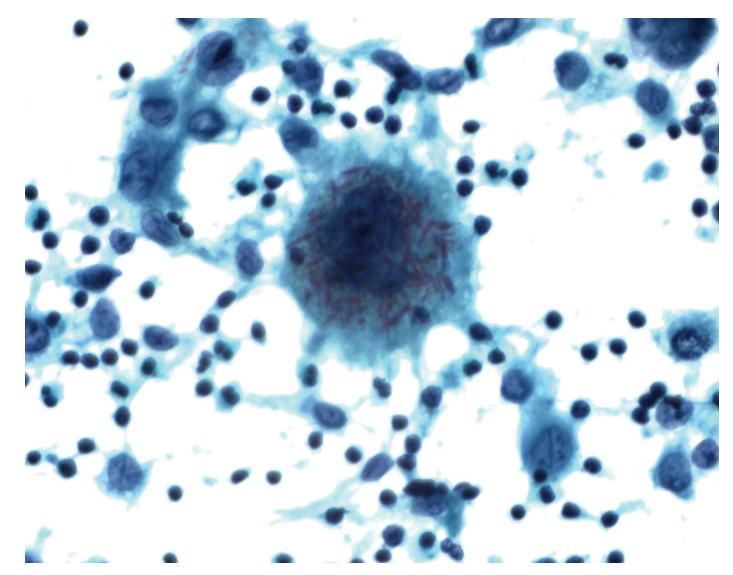

(b)

Figure 3: (a) Extracellular rhomboid Charcot-Leyden crystals (Papanicolaou stain, original magnification, x400). (b) Macrophages with several ingested Charcot-Leyden crystals (Papanicolaou stain, original magnification, $\mathrm{x} 400$ ).

and case series [16-31]. Study of these shows that cytology closely reflects histomorphology. Ancillary studies may not be always necessary for diagnosis in appropriate setting [32] .

The classical cytological features include high cellularity composed of sheets and many isolated LCs seen admixed with polymorphous population of numerous eosinophils, neutrophils, lymphocytes, plasma cells, multinucleated giant cells, and macrophages. The key to the diagnosis is to identify the LC through its characteristic features, namely, nuclear grooves and nuclear pseudoinclusions. They show variable degree of pleomorphism and mitotic activity $[17,18,22,26$, 29]. Presence of dendrite-like cytoplasmic processes in LCs is a rare but characteristic feature $[22,33,34]$. Sometimes the LCs are few or nuclear grooves not very prominent or lack cytopalsmic processes. Degree of eosinophil infiltration varies in different areas of $\mathrm{LCH}$ lesion and different organs, thus their number can vary from scant to abundant in cytology smears [22]. Their presence can help attract attention to the diagnosis. In our case, eosinophils were more abundant in lymph node smears as compared to skull lesions which had more of LCs and reactive histiocytes.

Presence of Charcot-Leyden crystals singly and in bunches within the macropahges, giant cells, and extarcellularly was a unique feature in our case and has been reported very rarely $[20,27,29,31]$. Charcot-Leyden crystals are crystalloids containing eosinophil membrane protein formed from rupture of eosinophil's granules. They indicate tissue eosinophilia and may help in drawing attention to the $\mathrm{LCH}$ diagnosis.

The diagnosis of LCH in our patient was made on the basis of FNA which showed characteristic (both common and rare) features of LCH. This was corroborated by characteristic radiology and clinical findings. In this case, CT showed lytic lesions in the skull bones having sharp borders with a punched-out appearance. Destruction of both the inner and outer tables results in a double-contour or bevelededge appearance which is a typical feature in the diagnosis of LCH $[35,36]$.
The cytologic diagnosis may be missed due to lack of familiarity with its cytological features among pathologists or due to the lack of characteristic cytological findings resulting from a sampling error. Therefore it is prudent on the part of the pathologist to consider this diagnosis only in an appropriate clinical and radiological setting. It is also necessary to be familiar with cytological features of other differential diagnoses.

In the present case, the most common differential diagnoses of skull lesions clinically included Ewing's sarcoma, non-Hodgkin lymphoma, and osteomyelitis. Ewing's sarcoma and non-Hodgkin lymphoma are characterized by monotonous population of small round blue cells. In acute osteomyelitis, the neutrophils form a prominent component. The reactive histiocytes are seen and can be easily distinguished due to the absence of distinctive features of LCs. Chronic osteomyeltis shows predominantly plasma cells and lymphocytes. Plasma cells and neutrophils are infrequent in LCH.

Sinus histiocytosis with massive lymphadenopathy (SHML) involves primarily the cervical nodes, but its histiocytes are morphologically quite different from those of LCH. In SHML, the histiocytes have abundant cytoplasm, exhibiting hematopoietic phagocytosis and prominent nucleoli [28].

Secondary hyperplasia of the LCs is associated with lymphomas, especially with Hodgkin's disease and lung tumors. Care should be taken to differentiate these hyperplastic Langerhans cells from atypical LCs of LCH. Rarely, LCH can be associated with another malignancy such as malignant lymphoma, leukemia, or metastatic neoplasm [37, 38]. These need to be excluded after a diligent search for malignant cells with obvious cytologic atypia in the smear. Malignancies with tumor cells commonly having nuclear grooves or pseudoinclusions should also be considered, such as malignant melanoma and papillary thyroid carcinoma.

LCs show positivity for S-100, PNA (peanut agglutinin), MHC class II, CD1a, and langerin (CD207) [2]. Our case 
showed positivity for S-100 protein. CD1a and langerin are not available in our lab. The Birbeck granule is their distinctive ultrastructural hallmark [2]. Electron microscopy was not performed in our patient and was not considered essential for diagnosis as also suggested by other authors [32].

Patients with apparently restricted $\mathrm{LCH}$ need careful staging of their disease to ensure that the lesions are not part of a more extensive process. FNA can be used to establish the extent of disease or recurrence of LCH [18]. In children with multiple swellings as in our case, FNA, being minimally invasive, is particularly suitable to sample all swellings in detecting the extent of involvement. For localized lesions in the skeletally immature patients, a simple, minimally invasive form of treatment with a low rate of complication is desirable. In view of this and the possibility of spontaneous resolution in localized disease, FNA alone could be used to confirm the diagnosis.

To conclude, the present case highlights the role of FNA in the diagnosis of the rare disease of $\mathrm{LCH}$ in a child with usual clinical presentation. The cytologic features of LCH are highly characteristic to suggest a diagnosis in an appropriate clinical setting with classical radiological findings. A high index of suspicion, awareness of common and rare cytological features of LCH, its differential diagnoses, and causes of diagnostic pitfalls is necessary. This can obviate the need of biopsy and electron microscopy. Immunochemistry if available can be performed on cell block.

\section{References}

[1] L. Buchmann, A. Emami, and J. L. Wei, "Primary head and neck Langerhans cell histiocytosis in children," Otolaryngology, vol. 135, no. 2, pp. 312-317, 2006.

[2] C. R. Shea and M. D. Boos, "Langerhan Cell Histiocytosis," 2009, http://emedicine.medscape.com/article/1100579-overview.

[3] L. Lichtenstein, "Histiocytosis X: integration of eosinophilic granuloma of bone, "Letterer-Siwe disease" and "Schuller Christian disease" as related manifestations of single nosologic entity," Archives of Pathology \& Laboratory Medicine, vol. 56, pp. 84-102, 1953.

[4] V. Broadbent, H. Gadner, D. M. Komp et al., "Histiocytosis syndromes in children: II. Approach to the clinical and laboratory evaluation of children with Langerhans cell histiocytosis," Medical and Pediatric Oncology, vol. 17, no. 6, pp. 492-495, 1989.

[5] B. A. Degar and B. J. Rollins, "Langerhans cell histiocytosis: malignancy or inflammatory disorder doing a great job of imitating one?" DMM Disease Models and Mechanisms, vol. 2, no. 9-10, pp. 436-439, 2009.

[6] L. Gong, W.-D. Zhang, Y.-H. Li et al., "Clonal status and clinicopathological features of langerhans cell histiocytosis," Journal of International Medical Research, vol. 38, no. 3, pp. 1099-1105, 2010.

[7] Y. Kawakubo, H. Kishimoto, Y. Sato et al., "Human cytomegalovirus infection in foci of Langerhans cell histiocytosis," Virchows Archiv, vol. 434, no. 2, pp. 109-115, 1999.

[8] R. M. Egeler, B. E. Favara, M. Van Meurs, J. D. Laman, and E. Claassen, "Differential in situ cytokine profiles of Langerhanslike cells and T cells in Langerhans cell histiocytosis: abundant expression of cytokines relevant to disease and treatment," Blood, vol. 94, no. 12, pp. 4195-4201, 1999.

[9] U. A. By, E. Tani, U. Andersson, and J. I. Henter, "Tumor necrosis factor, interleukin 11, and leukemia inhibitory factor produced by langerhans cells in langerhans cell histiocytosis," Journal of Pediatric Hematology/Oncology, vol. 26, no. 11, pp. 706-711, 2004.

[10] C. E. T. da Costa, K. Szuhai, R. Van Eijk et al., "No genomic aberrations in langerhans cell histiocytosis as assessed by diverse molecular technologies," Genes Chromosomes and Cancer, vol. 48, no. 3, pp. 239-249, 2009.

[11] K. Windebank and V. Nanduri, "Langerhans cell histiocytosis," Archives of Disease in Childhood, vol. 94, no. 11, pp. 904-908, 2009.

[12] J. W. Williams and R. F. Dorfman, "Lymphadenopathy as the initial manifestation of histiocytosis X," American Journal of Surgical Pathology, vol. 3, no. 5, pp. 405-421, 1979.

[13] R. L. Katz, E. G. Silva, and L. A. DeSantos, "Diagnosis of eosinophilic granuloma of bone by cytology, histology, and electron microscopy of transcutaneous bone-aspiration biopsy," Journal of Bone and Joint Surgery-Series A, vol. 62, no. 8, pp. 1284-1290, 1980.

[14] B. E. Favara and R. Jaffe, "The histopathology of Langerhans cell histiocytosis," British Journal of Cancer, vol. 70, no. 23, supplement, pp. S17-S23, 1994.

[15] J. Wang, X. Wu, and Z.-J. Xi, "Langerhans cell histiocytosis of bone in children: a clinicopathologic study of 108 cases," World Journal of Pediatrics, vol. 6, no. 3, pp. 255-259, 2010.

[16] L. J. Layfield and S. Bhuta, "Fine-needle aspiration cytology of histiocytosis X: a case report," Diagnostic Cytopathology, vol. 4, no. 2, pp. 140-143, 1988.

[17] J.-P. Musy, L. Ruf, and I. Ernerup, "Cytopathologic diagnosis of an eosinophilic granuloma of bone by needle aspiration biopsy," Acta Cytologica, vol. 33, no. 5, pp. 683-685, 1989.

[18] T. Elsheikh, J. F. Silverman, P. E. Wakely Jr., C. T. Holbrook, and V. V. Joshi, "Fine-needle aspiration cytology of Langerhans' cell histiocytosis (eosinophilic granuloma) of bone in children," Diagnostic Cytopathology, vol. 7, no. 3, pp. 261-266, 1991.

[19] J. K. Granger and H. Y. Houn, "Eosinophilic granuloma of lymph node: case report with cytohistologic, immunohistochemical, and flow cytometric observations," Diagnostic Cytopathology, vol. 7, no. 4, pp. 402-407, 1991.

[20] P. Van Heerde and R. M. Egeler, "The cytology of Langerhans cell histiocytosis (histiocytosis X)," Cytopathology, vol. 2, no. 3, pp. 149-158, 1991.

[21] N. Shabb, C. V. Fanning, C. H. Carrasco et al., "Diagnosis of eosinophilic granuloma of bone by fine-needle aspiration with concurrent institution of therapy: a cytologic, histologic, clinical, and radiologic study of 27 cases," Diagnostic Cytopathology, vol. 9, no. 1, pp. 3-12, 1993.

[22] M. Akhtar, M. A. Ali, M. Bakry, K. Sackey, and R. Sabbah, "Fine-needle aspiration biopsy of Langerhans histiocytosis (histiocytosis- X)," Diagnostic Cytopathology, vol. 9, no. 5, pp. 527-533, 1993.

[23] P. R. N. Kirchgraber, M. G. Weaver, B. M. Arafah, and F. W. Abdul-Karim, "Fine needle aspiration cytology of Langerhans cell histiocytosis involving the thyroid: a case report," Acta Cytologica, vol. 38, no. 1, pp. 101-106, 1994.

[24] D. K. Das and N. C. Nayak, "Diagnosis of Langerhans cell histiocytosis by fine needle aspiration cytology," Acta Cytologica, vol. 39, no. 6, pp. 1260-1263, 1995. 
[25] P. Demille, R. Weihing, and C. C. J. Sun, "Intraoperative diagnosis of osseous eosinophilic granuloma by touch preparation: report of two cases with immunohistochemistry and electron microscopy," Diagnostic Cytopathology, vol. 14, no. 1, pp. 6871, 1996.

[26] Z. Pohar-Marinsek and M. Us-Krasovec, "Cytomorphology of Langerhans cell histiocytosis," Acta Cytologica, vol. 40, no. 6, pp. 1257-1264, 1996.

[27] J. S. Lee, M. C. Lee, C. S. Park, and S. W. Juhng, "Fine needle aspiration cytology of Langerhans cell histiocytosis confined to lymph nodes: a case report," Acta Cytologica, vol. 41, no. 6, pp. 1793-1796, 1997.

[28] S. Kakkar, K. Kapila, and K. Verma, "Langerhans cell histiocytosis in lymph nodes cytomorphologic diagnosis and pitfalls," Acta Cytologica, vol. 45, no. 3, pp. 327-332, 2001.

[29] P. V. Kumar, A. Mousavi, M. Karimi, and G. R. Bedayat, "Fine needle aspiration of Langerhans cell histiocytosis of the lymph nodes: a report of six cases," Acta Cytologica, vol. 46, no. 4, pp. 753-756, 2002.

[30] L.-Y. Lee, C.-J. Kang, Y.-Y. Hsieh, and S. Hsueh, "Diagnosis of nodal Langerhans cell histiocytosis by fine needle aspiration cytology," Chang Gung Medical Journal, vol. 28, no. 10, pp. 735-739, 2005.

[31] T. K. Kobayashi, M. Ueda, T. Nishino et al., "Langerhans cell histiocytosis of the skull on cytologic squash preparations," Diagnostic Cytopathology, vol. 35, no. 3, pp. 154-157, 2007.

[32] S. E. Kilpatrick, "Fine needle aspiration biopsy of Langerhans cell histiocytosis of bone: are ancillary studies necessary for a "definitive" diagnosis?" Acta Cytologica, vol. 42, no. 3, pp. 820823, 1998.

[33] P. Malhotra, R. Tandon, N. Singh, V. K. Arora, and A. Bhatia, "Cytoplasmic processes: a distinct cytomorphologic feature of Langerhans cell histiocytosis," Acta Cytologica, vol. 49, no. 5, pp. 580-582, 2005.

[34] G. Jayaram, "Cytoplasmic processes as a diagnostic aid in langerhans cell histiocytosis," Acta Cytologica, vol. 51, no. 5, pp. 833-834, 2007.

[35] R. Hermans, B. De Foer, M. H. Smet et al., "Eosinophilic granuloma of the head and neck: CT and MRI features in three cases," Pediatric Radiology, vol. 24, no. 1, pp. 33-36, 1994.

[36] H. C. Chen, W. C. Shen, D. Y. Chou, and I. P. Chiang, "Langerhans cell histiocytosis of the skull complicated with an epidural hematoma," American Journal of Neuroradiology, vol. 23, no. 3, pp. 493-495, 2002.

[37] R. M. Egeler, J. P. Neglia, M. Arico et al., "The relation of Langerhans cell histiocytosis to acute leukemia, lymphomas, and other solid tumors. The LCH-Malignancy Study Group of the Histiocyte Society," Hematology/Oncology Clinics of North America, vol. 12, no. 2, pp. 369-378, 1998.

[38] R. M. Egeler, J. P. Neglia, D. M. Puccetti, C. A. Brennan, and M. E. Nesbit, "Association of Langerhans cell histiocytosis with malignant neoplasms," Cancer, vol. 71, no. 3, pp. 865-873, 1993. 


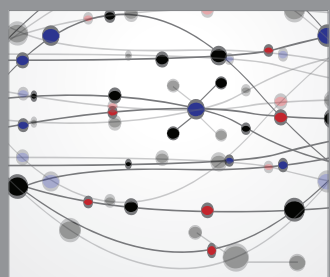

The Scientific World Journal
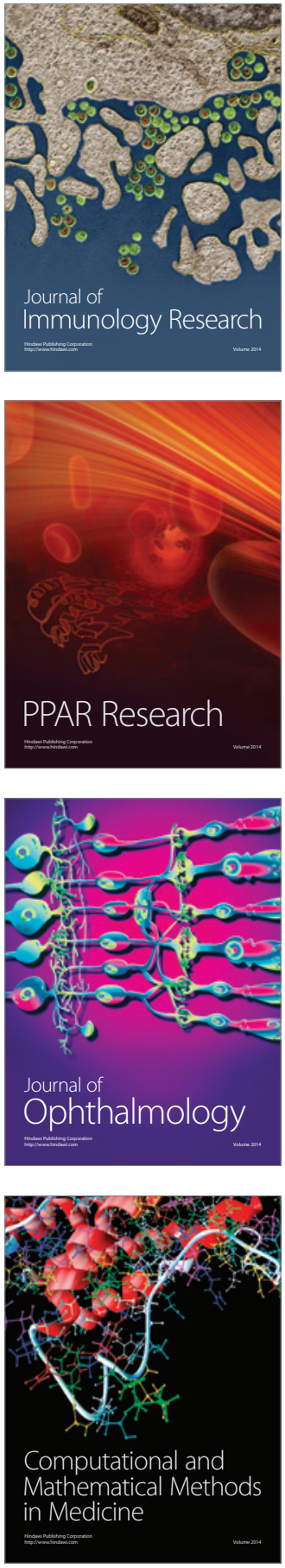

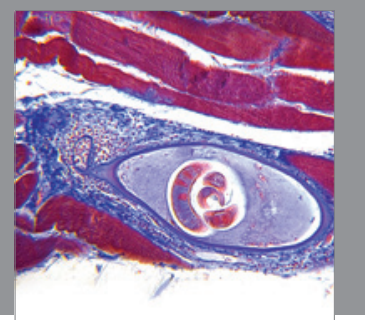

Gastroenterology

Research and Practice
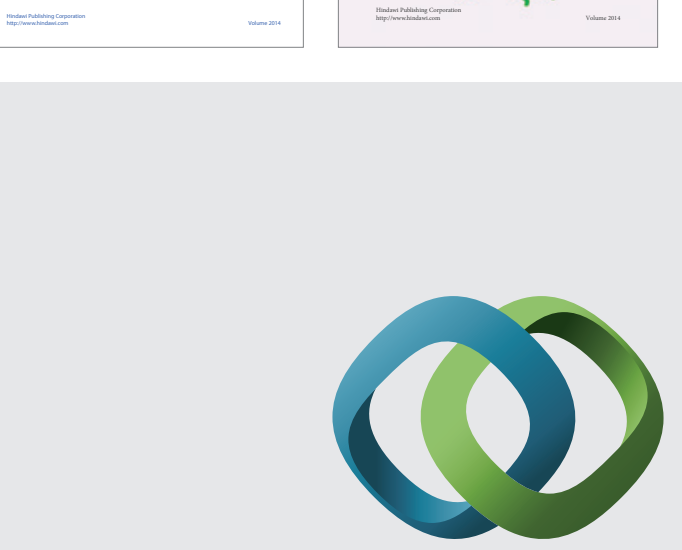

\section{Hindawi}

Submit your manuscripts at

http://www.hindawi.com
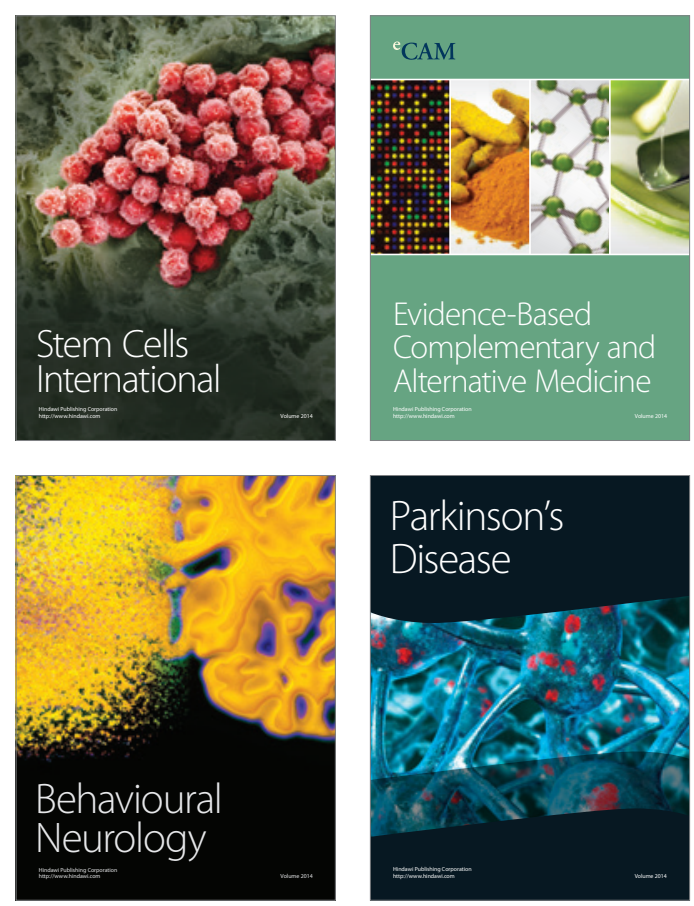

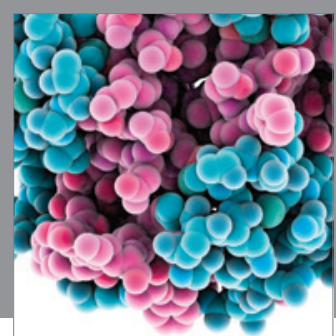

Journal of
Diabetes Research

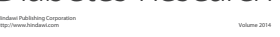

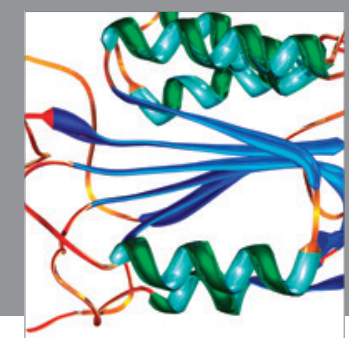

Disease Markers
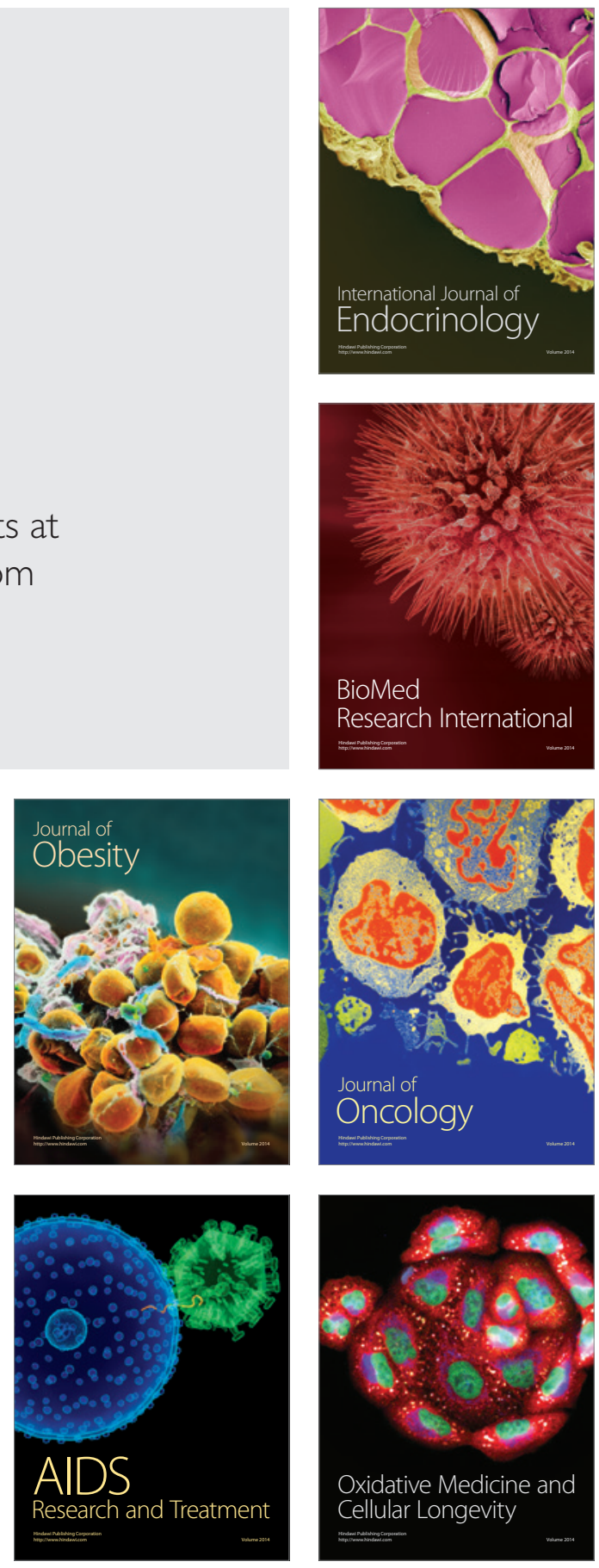